\title{
Brain metastases originating in breast cancer: clinical- pathological analysis and immunohistochemical profile
}

\author{
AnCa Sava ${ }^{1,2)}$, Claudia Florida Costea ${ }^{3)}$, RuXandRa Vatavu4), Mihaela GRigore ${ }^{5,6)}$, \\ MiHAELA DANA TURLIUC ${ }^{3,7)}$, GABRIELA FLORENTA DUMITRESCU2), LUCIAN EVA ${ }^{7)}$, \\ ANDREI GHEORGHE MARIUS MOTOC ${ }^{8)}$, CRISTINEL IONEL STAN ${ }^{1)}$, LIVIU CiPRIAN GAVRIL ${ }^{1}$, \\ SADIYE IOANA SCRIPCARIU6)
}

\author{
1) Department of Morphofunctional Sciences I, Faculty of Medicine, Grigore T. Popa University of Medicine and Pharmacy, \\ Iaşi, Romania \\ 2) Laboratory of Pathology, Prof. Dr. Nicolae Oblu Emergency Clinical Hospital, Iaşi, Romania \\ 3) Department of Surgery II, Faculty of Medicine, Grigore T. Popa University of Medicine and Pharmacy, Iaşi, Romania \\ 4) PhD Student, Doctoral School, Grigore T. Popa University of Medicine and Pharmacy, Iaşi, Romania \\ ${ }^{5)}$ Department of Obstetrics and Gynecology, Faculty of Medicine, Grigore T. Popa University of Medicine and Pharmacy, \\ Iaşi, Romania \\ ${ }^{6)}$ Clinic of Gynecology, Cuza Vodă Hospital of Obstetrics and Gynecology, Iaşi, Romania \\ 7) $2^{\text {nd }}$ Clinic of Neurosurgery, Prof. Dr. Nicolae Oblu Emergency Clinical Hospital, Iaşi, Romania \\ ${ }^{8)}$ Department of Anatomy and Embryology, Victor Babeş University of Medicine and Pharmacy, Timişoara, Romania
}

\begin{abstract}
The incidence of brain metastases (BMs) originating in breast cancer $(\mathrm{BC})$ is increasing due to advances in imaging techniques, which can detect such events early, and due to new therapies that can ensure longer survival. We performed a retrospective study on patients with BMs originating in BC and receiving surgical treatment in Neurosurgery Clinics of Prof. Dr. Nicolae Oblu Emergency Clinical Hospital, laşi, Romania, from January 2018 to December 2019. We identified 10 consecutive patients who underwent a craniotomy for a BMs originating in a BC (either for diagnostic purpose or with therapeutic intent). Clinicopathological data were collected from the electronic medical record and included the patient demographics (age at diagnosis of their BM), morphological characteristics of BM [location, cytopathological features, histopathological (HP) subtype, and immunohistochemical features, i.e., cytokeratin 5/6 (CK5/6), mammaglobin, estrogen receptor (ER), progesterone receptor (PR), and Ki67 labeling index (LI)], and time from BC diagnosis to BM diagnosis. Ninety percent of patients were in their sixth and seventh decades of life, with a median age of 57.9 years (47-65 years). Median time from BC diagnosis to BM was 34.42 months. Fifty percent of BMs were located in the parietal lobes, and $70 \%$ of all cases have multiple $(\geq 2)$ BMs. All cases $(100 \%)$ had a cytopathological examination, showing a hypercellular pattern, with poorly cohesive clusters of mild or pleomorphic cells, with nuclei with homogeneously distributed fine granular chromatin membrane, and with small or enlarged and irregular nucleoli. The dominant histopathology was invasive breast carcinoma of no special type (IBC-NST) (70\%), but we also identified specific subtypes, i.e., tubular carcinoma (TC) $(20 \%)$ and invasive micropapillary carcinoma (IMPC) (10\%). Correlating location with HP subtypes of BMs from BC, IBC-NST and IMPC were located mostly in parietal lobes, and TC developed only in the occipital lobe. We found three patterns of immunostaining: (i) CK5/6 +/-, mammaglobin+, ER+, PR-, which was much more characteristic for IBC-NST; (ii) CK5/6-, mammaglobin+, ER-, PR-, being identified in tubular breast carcinoma; (iii) $\mathrm{CK} 5 / 6$ +/-, mammaglobin-, ER-, PR-, which were revealed by invasive micropapillary breast carcinoma. Our study revealed the fact that $\mathrm{BMs}$ originating in $\mathrm{BC}$ show heterogeneity of hormone receptor status, although morphologically there is not so much diversity. We also found a very variable Ki67 LI, which correlated especially with the morphological subtype.
\end{abstract}

Keywords: breast cancer, brain metastases, mammaglobin, estrogen receptor, progesterone receptor, Ki67 labeling index.

\section{Introduction}

Brain metastases (BMs) originating in breast cancer (BC) represent $24 \%$ of all metastases located at this level and their percentage is increasing due to advances in imaging techniques, which can detect such events early, and due to new therapies that can ensure longer survival [1]. Lin et al. (2008) even reported a much higher percentage, which can range from $25 \%$ to $46 \%$ in patients with metastatic triple-negative BC (TNBC) [2].

$\mathrm{BC}$ is raising worldwide, this neoplasm being the second leading cause of cancer death worldwide after lung cancer. If only female mortality from cancer is considered, then $\mathrm{BC}$ comes first [3].

According to World Health Organization (WHO) statistics, $\mathrm{BC}$ ranks first as the most common cause of death from a female malignancy, accounting for $17.0 \%$ of the 20200 deaths in 2014. Among the female population in Romania, the incidence of $\mathrm{BC}$ is twice higher than that of colorectal or cervical cancer, registering a number of 8981 cases compared to 4496 and 4343 cases, respectively [4].

In the past, the brain was a relatively rare site of metastasis of a BC, probably due to lack of proper treatment.

This is an open-access article distributed under the terms of a Creative Commons Attribution-NonCommercial-ShareAlike 4.0 International Public License, which permits unrestricted use, adaptation, distribution and reproduction in any medium, non-commercially, provided the new creations are licensed under identical terms as the original work and the original work is properly cited. 
However, with the refinement of the treatment, especially the chemotherapeutic one, there are estimations that $10-16 \%$ of women with BC can develop symptomatic BMs at one moment of the course of their neoplasia [5]. Symptoms and sign due to BMs depend on the location of the lesion. These patients can manifest various symptoms, such as seizures, visual disturbance, focal neurological dysfunction, altered mental state or stroke [6]. Rarely, BMs could be detected as the first site of metastasizing of a $\mathrm{BC}$, but more often BM usually occurs late during $\mathrm{BC}$, after first developing in lung, liver, or bones. The prognosis of these patients is poor, the average one-year survival being about $20 \%$.

\section{Aim}

The aim of this study was to analyze the clinicalpathological features and the immunoprofile of BMs originating in $\mathrm{BC}$ to identify the risk factors for the occurrence of these neoplasias.

\section{口 Patients, Materials and Methods}

A retrospective study on patients with BMs originating in $\mathrm{BC}$ and receiving surgical treatment in Neurosurgery Clinics of Prof. Dr. Nicolae Oblu Emergency Clinical Hospital, Iaşi, Romania, from January 2018 to December 2019 was performed. We identified 10 consecutive patients who underwent a craniotomy for a BMs originating in a BC (either for diagnostic purpose or with therapeutic intent).

Clinicopathological data were collected from the electronic medical records and included the patient demographics (age at diagnosis of their BM), morpho- logical characteristics of BM [location, cytopathological features, histopathological (HP) subtype, and immunohistochemical (IHC) features], and time from BC diagnosis to $\mathrm{BM}$ diagnosis. We only included women with pathologically diagnosed BMs from $\mathrm{BC}$.

Cytopathological exam was realized on intra-operative smears using small specimens obtained during surgical intervention. We placed a small sample $\left(1-2 \mathrm{~mm}^{3}\right)$ of the biopsy specimen on one edge of a clean, dry and labeled slide and crushed it with the edge of another clean, dry and labeled slide with enough pressure to spread the bioptic tissue into a thin film on both slides. Then both slides were immediately stained with $1 \%$ Toluidine Blue staining. In all $10(100 \%)$ cases the cytological diagnosis was consistent with a BM.

The remaining biopsy was submitted for formalin fixation. We used $4 \mu \mathrm{m}$ HP sections, which were obtained from cutting at microtome the paraffin-embedded tissue blocks, and we stained them with Hematoxylin-Eosin (HE) staining. A pathologist established the diagnosis of a BM from a previous BC.

For this study, all slides and blocks from all these 10 BMs were retrieved. Two pathologists reviewed all HEstained sections and tumors were classified according to the WHO Classification of Tumors of the Breast (2019) [7]. Representative sections of BM were used for IHC stainings, to identify the origin of the $\mathrm{BM}$ in a previous $\mathrm{BC}$ [anti-cytokeratin 5/6 (CK5/6) and anti-mammaglobin antibodies], the immunoexpression of estrogen receptor (anti-ER antibody) and progesterone receptor (anti-PR antibody), but also the tumor proliferative activity (antiKi67 antibody) (Table 1).

Table 1 - Manufacturer descriptions of the antibodies we used in this study

\begin{tabular}{ccccccc}
\hline Antibody & Manufacturer & Clone & Antigen retrieval & Class & Dilution & Cellular location \\
\hline Anti-CK5/6 & Bio SB, USA & EP24/EP67 & Citrate, $\mathrm{pH} \mathrm{6}$ & Rabbit monoclonal & $1: 100$ & Cytoplasm \\
\hline Anti-mammaglobin & Bio SB, USA & EP249 & Citrate, $\mathrm{pH} 6$ & Rabbit monoclonal & Prediluted & Cytoplasm \\
\hline Anti-ER & Bio SB, USA & RBT11 & Citrate, $\mathrm{pH} 6$ & Rabbit monoclonal & Prediluted & Nucleus \\
\hline Anti-PR & Dako, USA & PgR636 & Citrate, $\mathrm{pH} \mathrm{6}$ & Mouse monoclonal & $1: 50$ & Nucleus \\
\hline Anti-Ki67 & Dako, Denmark & MIB-1 & Citrate, $\mathrm{pH} \mathrm{6}$ & Mouse monoclonal & $1: 75$ & Nucleus \\
\hline
\end{tabular}

CK5/6: Cytokeratin 5/6; ER: Estrogen receptor; PR: Progesterone receptor.

IHC reactions were performed using the EnVision ${ }^{\mathrm{TM}}+$ Dual Link System-Horseradish Peroxidase (HRP) (Dako, Denmark) for mammaglobin, cytokeratin 5/6 (CK5/6) and ER, and UltraVision Quanto Detection System-HRP (Thermo Scientific, UK) for PR and Ki67 labeling index (LI), according to manufacturers' recommendations. Antigen retrieval was realized with sodium citrate buffer, $\mathrm{pH} 6$, using a water bath at $95^{\circ} \mathrm{C}$. In the end, 3,3'-Diaminobenzidine (DAB) was used as chromogen and Mayer's Hematoxylin for nuclear counterstaining against nuclear staining. Normal breast tissue was used as positive controls. IHC reactions were considered positive only when a brown staining of the cytoplasm (in case of anti-CK5/6 and anti-mammaglobin antibodies) or of the nucleus (in case of anti-ER, anti-PR, and anti-Ki67 antibodies) were obtained.

We calculated the mean Ki67 LI by counting both positive and negative nuclei in 10 different microscopic fields using a $40 \times$ objective. Firstly, we calculated the percentage of positive cells in each field, and then we used the arithmetic mean of the 10 previously recorded values to found out the mean Ki67 LI.
Data was first summarized using standard descriptive statistics and frequency tabulation.

\section{ㅁ Results}

Ten female patients with solitary or multiple BMs from BC underwent a craniotomy during the study period. Patients and tumor characteristics included in the study are summarized in Table 2. Ninety percent of patients were diagnosed with BMs from $\mathrm{BC}$ between the ages of 50-69 years (Figure 1a), with a median age of 57.9 years (47-65 years).

Median time from $\mathrm{BC}$ diagnosis to $\mathrm{BM}$ was 34.42 months, if we analyzed only the cases with BMs previously diagnosed with BC. For the whole series, however, because in three $(25 \%)$ of all cases studied, BC was diagnosed synchronously with $\mathrm{BMs}$, median time from $\mathrm{BC}$ diagnosis to $\mathrm{BM}$ was 24.1 months. Female patients older than 60 years at $\mathrm{BM}$ diagnosis had a $\mathrm{BC}$ diagnosis more than 20 months ago. Patients younger than 60 years were diagnosed with $\mathrm{BM}$ after less than 20 months since the $\mathrm{BC}$ diagnosis (Figure 1b). 
Figure 1 - (a and b) Correlation between age of the patients and the period of time from breast carcinoma $(B C)$ diagnosis to brain metastasis (BM) diagnosis.
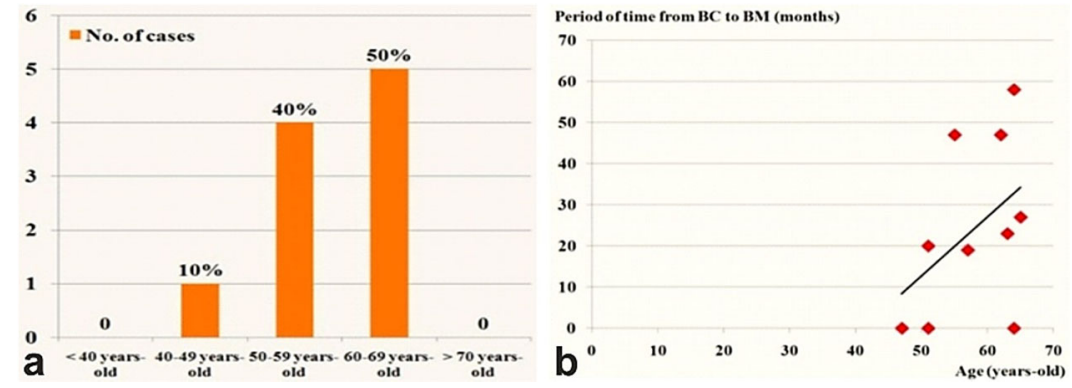

Table 2 - Clinical and pathological characteristics of patients with BMs from BC

\begin{tabular}{|c|c|c|c|c|c|c|c|c|}
\hline $\begin{array}{l}\text { Case } \\
\text { No. }\end{array}$ & $\begin{array}{c}\text { A } \\
\text { [years] }\end{array}$ & nBMs & locBMs & Cytological features & HistoBM & $\mathrm{pBC}$ & TreatBC & $\begin{array}{c}\text { tBMs } \\
\text { [months] }\end{array}$ \\
\hline 1. & 51 & 3 & RPL & $\begin{array}{l}\text { High cellularity, tumor pleomorphic epithelial cells } \\
\text { with small nucleoli }\end{array}$ & $\mathrm{TC}$ & No & - & - \\
\hline 2. & 55 & 1 & RPL & $\begin{array}{c}\text { Moderate cellularity, tumor epithelial cells with } \\
\text { moderate pleomorphism }\end{array}$ & IBC-NST & Yes & $\begin{array}{c}\mathrm{M} \\
\mathrm{Ch}, \mathrm{RT}\end{array}$ & 47 \\
\hline 3. & 47 & 2 & LCer & $\begin{array}{c}\text { High cellularity pleomorphic epithelial cells, small } \\
\text { nucleoli }\end{array}$ & IBC-NST & No & - & - \\
\hline 4. & 64 & 1 & RPL & Tumor epithelial cells with moderate pleomorphism & IBC-NST & Yes & Ch, RT & 58 \\
\hline 5. & 63 & 2 & $\mathrm{ROL}$ & $\begin{array}{c}\text { High cellularity pleomorphic epithelial cells, small } \\
\text { nucleoli }\end{array}$ & $\mathrm{TC}$ & Yes & M & 23 \\
\hline 6. & 57 & 3 & RCer & $\begin{array}{l}\text { High cellularity pleomorphic epithelial cells, small } \\
\text { nucleoli }\end{array}$ & IBC-NST & Yes & Ch, RT & 19 \\
\hline 7. & 62 & 1 & LPL & $\begin{array}{l}\text { Markedly pleomorphic ductal cells with enlarged, } \\
\text { irregular nuclei, large and prominent nucleoli and } \\
\text { mitosis }\end{array}$ & IBC-NST & Yes & $\begin{array}{c}M \\
\text { Ch, RT }\end{array}$ & 47 \\
\hline 8. & 65 & 3 & LPL & $\begin{array}{l}\text { Tumor cells with light cytoplasm and eccentric } \\
\text { nuclei with fine granular chromatin and nucleoli }\end{array}$ & IMPC & Yes & $\begin{array}{c}\mathrm{M} \\
\mathrm{Ch}, \mathrm{RT}\end{array}$ & 27 \\
\hline 9. & 64 & 4 & LOL & $\begin{array}{l}\text { High cellularity moderate cellular pleomorphism, } \\
\text { and small nucleoli }\end{array}$ & IBC-NST & No & - & - \\
\hline 10. & 51 & 3 & LPL & $\begin{array}{l}\text { High cellularity pleomorphic epithelial cells, small } \\
\text { nucleoli }\end{array}$ & IBC-NST & Yes & $\begin{array}{c}\mathrm{M} \\
\mathrm{Ch}, \mathrm{RT} \\
\end{array}$ & 20 \\
\hline
\end{tabular}

A: Patient's age at the time of admission to neurosurgery; BC: Breast carcinoma; BM: Brain metastasis; Ch: Chemotherapy; HistoBM: Histopathological subtype of brain metastasis; IBC-NST: Invasive breast carcinoma of no special type; IMPC: Invasive micropapillary carcinoma; LCer: Left cerebellum; locBMs: Location of brain metastases; LOL: Left occipital lobe; LPL: Left parietal lobe; M: Mastectomy; nBMs: No. of brain metastases; pBC: Previous known breast carcinoma; PL: Parietal lobe; RCer: Right cerebellum; ROL: Right occipital lobe; RPL: Right parietal lobe; RT: Radiotherapy; tBMs: Time to brain metastases (the period of time between the diagnosis of breast carcinoma and the diagnosis of brain metastases); TC: Tubular carcinoma; TreatBC: Type of treatment for breast carcinoma.

Our results showed that $\mathrm{BC}$ metastasized into the cerebellum (two cases, 20\%), right parietal lobe (two cases, $20 \%$ ), left parietal lobe (three cases, 30\%), right occipital lobe (two cases, 20\%), and left occipital lobe (one case, $10 \%$ ) (Figure 2). Considering the area where BC metastasized, the supratentorial area was most often involved compared with infratentorial area ( $80 \%$ versus $20 \%)$.

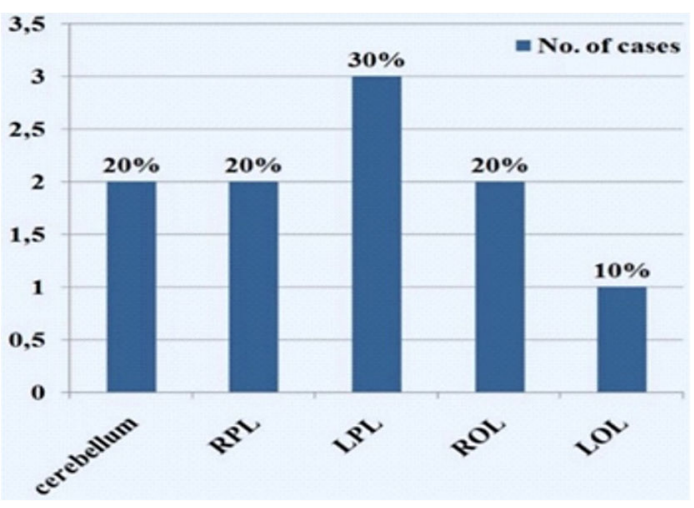

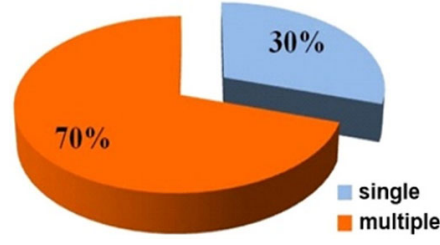

Thirty percent of patients were synchronously diagnosed with BMs and BC. Forty percent of patients were treated with mastectomy, chemotherapy, and radiotherapy, 20\% were treated with chemotherapy and radiotherapy, and only one case suffered a mastectomy. Thirty percent of patients were not known as having $\mathrm{BC}$ until the diagnosis of their BM.

All cases $(100 \%)$ with BM originating in a $\mathrm{BC}$ had a cytopathological examination, showing a hypercellular pattern, with poorly cohesive clusters of mild or pleomorphic cells, with nuclei with homogeneously distributed fine granular chromatin membrane, and with small or enlarged and irregular nucleoli (Figure 5, a-f). All cases $(100 \%)$ could be intra-operative diagnosed as having a BM from a carcinoma.

The dominant histopathology of the BMs from BC was invasive carcinoma of no special type (IBC-NST) (70\%), but we also identified specific subtypes, i.e., tubular carcinoma (TC) $(20 \%)$ and invasive micropapillary carcinoma (IMPC) (10\%) (Figure 6; Figure 7, a-d).

Figure 3 - Distribution of their number. BCBM: Breast cancer brain metastasis.
$70 \%$ patients had multiple $(\geq 2) \mathrm{BMs}$, and only $30 \%$ presented a single BM (Figure 3; Figure 4, a-d).
Figure 2 - Distribution of BCBM cases according to location. BCBM: Breast cancer brain metastasis,

LOL: Left occipital lobe; LPL: Left parietal lobe; ROL: Right occipital lobe; RPL: Right parietal lobe.

At the time of presentation to our Neurosurgery Clinics, 
Correlating location with HP subtypes of BMs from $\mathrm{BC}, 4 / 7$ cases of IBC-NST were located in parietal lobes, $1 / 7$ cases in occipital lobes and 2/7 cases in cerebellum; the two cases of TC developed only in the occipital lobe, and the only IMPC is identified only in the parietal lobe (Figure 8).

The IHC stainings are shown in Table 3 . We found three immunostaining patterns: (i) CK5/6 +/-, mammaglobin+, $\mathrm{ER}+, \mathrm{PR}-$, which was much more characteristic for IBCNST; (ii) CK5/6-, mammaglobin+, ER-, PR-, being identified in tubular breast carcinoma; (iii) CK5/6 +/-, mammaglobin-, ER-, PR-, which were revealed by invasive micropapillary breast carcinoma (Figures 9-11/a-e).

\begin{tabular}{ccc}
\multicolumn{3}{l}{$\begin{array}{l}\text { Table } 3 \text { - Immunopositivity of tumor cells for antibodies } \\
\text { used in this study }\end{array}$} \\
\cline { 2 - 3 } & Positive & Negative \\
\hline Mammaglobin & $70 \%$ & $3 \%$ \\
\hline CK5/6 & $20 \%$ (focal) & $80 \%$ \\
\hline PR & $20 \%$ & $80 \%$ \\
\hline ER & $40 \%$ & $60 \%$ \\
\hline Ki67 LI & $\begin{array}{c}100 \% \text { (its value ranges } \\
\text { from 3\% to 28\%) }\end{array}$ & - \\
\hline
\end{tabular}

CK5/6: Cytokeratin 5/6; ER: Estrogen receptor; LI: Labeling index; PR: Progesterone receptor.
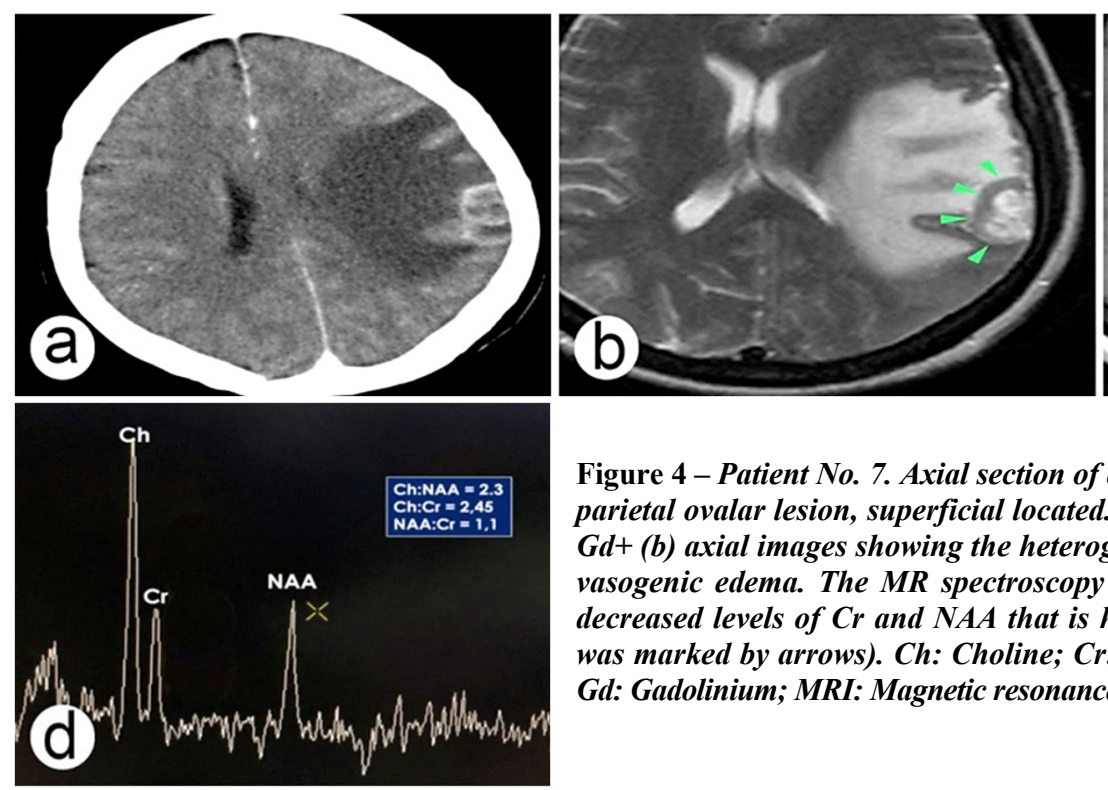

Figure 4 - Patient No. 7. Axial section of enhanced CT of the brain suggests a left parietal ovalar lesion, superficial located. MRI T2-weighted (a) and T1-weighted $G d+(b)$ axial images showing the heterogeneous lesion surrounded by important vasogenic edema. The MR spectroscopy (d) revealed elevated levels of Ch and decreased levels of $\mathrm{Cr}$ and $\mathrm{NAA}$ that is highly suggestive of malignancy (tumor was marked by arrows). Ch: Choline; Cr: Creatine; CT: Computed tomography; Gd: Gadolinium; MRI: Magnetic resonance (MR) imaging; NAA: N-acetylaspartate.
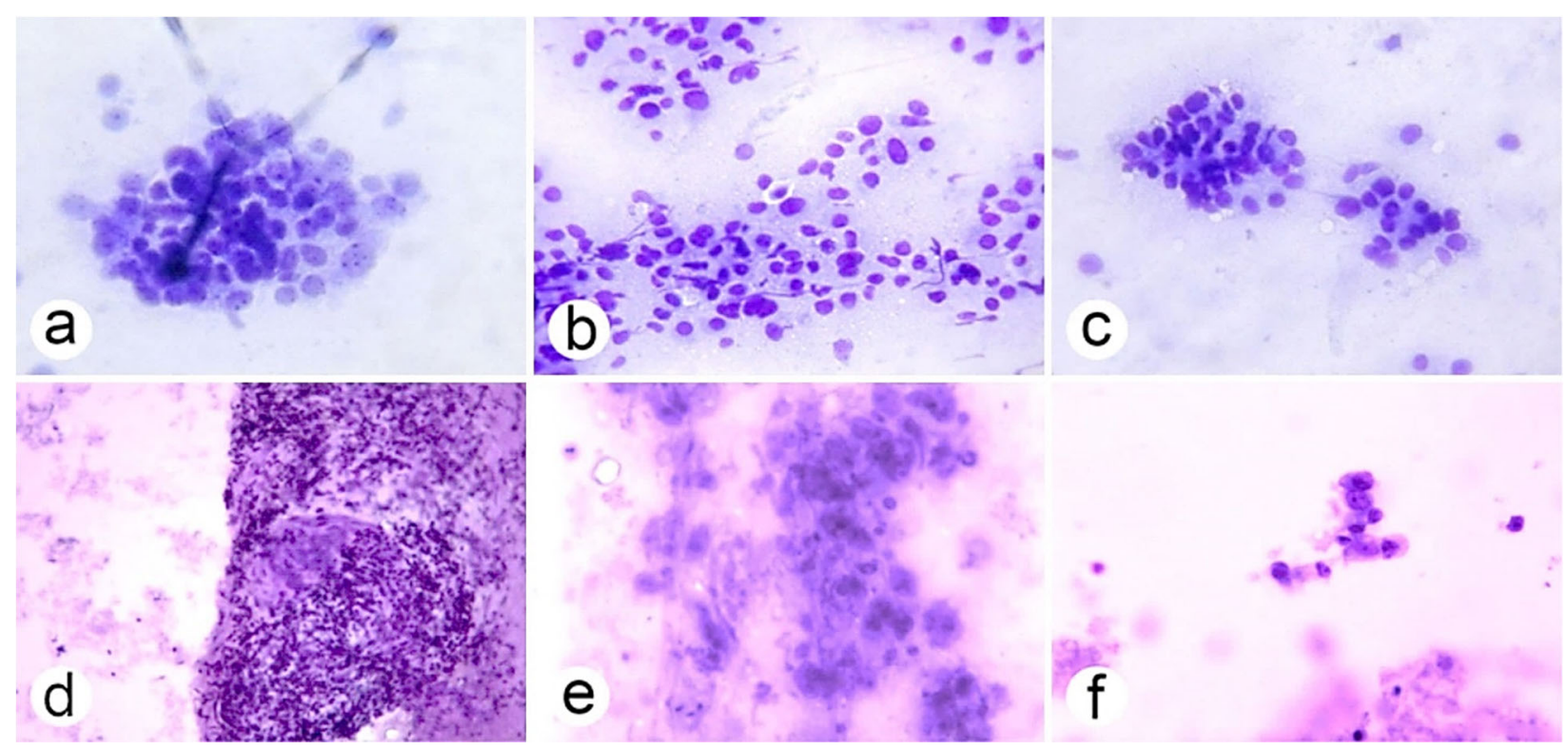

Figure 5 - Cytopathological features of brain metastases from breast carcinoma on intraoperative smear: (a) Patient No. 3 - high cellularity in a loosely cohesive cluster of mild pleomorphic ductal cells with nuclei showing homogeneously distributed fine granular chromatin membrane and small nucleoli; (b) Patient No. 9 - high cellularity with loosely cohesive clusters as well as single tumoral cells, with moderate pleomorphism, slightly irregular nuclear membrane, and small nucleoli; (c) Patient No. 1 - on a clear background, there are clusters and single tumoral ductal cells, with mild pleomorphism, light cytoplasm, regular nuclear membrane and indistinct nucleoli; (d) Patient No. 4 - a cluster of tumoral ductal cells amid the cells of the densely granular layer of the cerebellum; (e) Patient No. 7 - hypercellular pattern, with poorly cohesive clusters of markedly pleomorphic ductal cells with enlarged, irregular nuclei, presenting finely granular chromatin and large and prominent nucleoli and mitosis; the background showed granular debris; (f) Patient No. 8 - low cellular pattern with linear cell arrangements. Tumoral cells showed light cytoplasm and eccentric nuclei with fine granular chromatin and nucleoli. Toluidine Blue staining: $(a-c$, e and f) $\times 400 ;(d) \times 200$. 
Figure 6 - Histological subtypes of brain metastases from breast cancer. IBC-NST: Invasive breast carcinoma of no special type; IMPC: Invasive micropapillary breast carcinoma; TC: Tubular breast carcinoma.
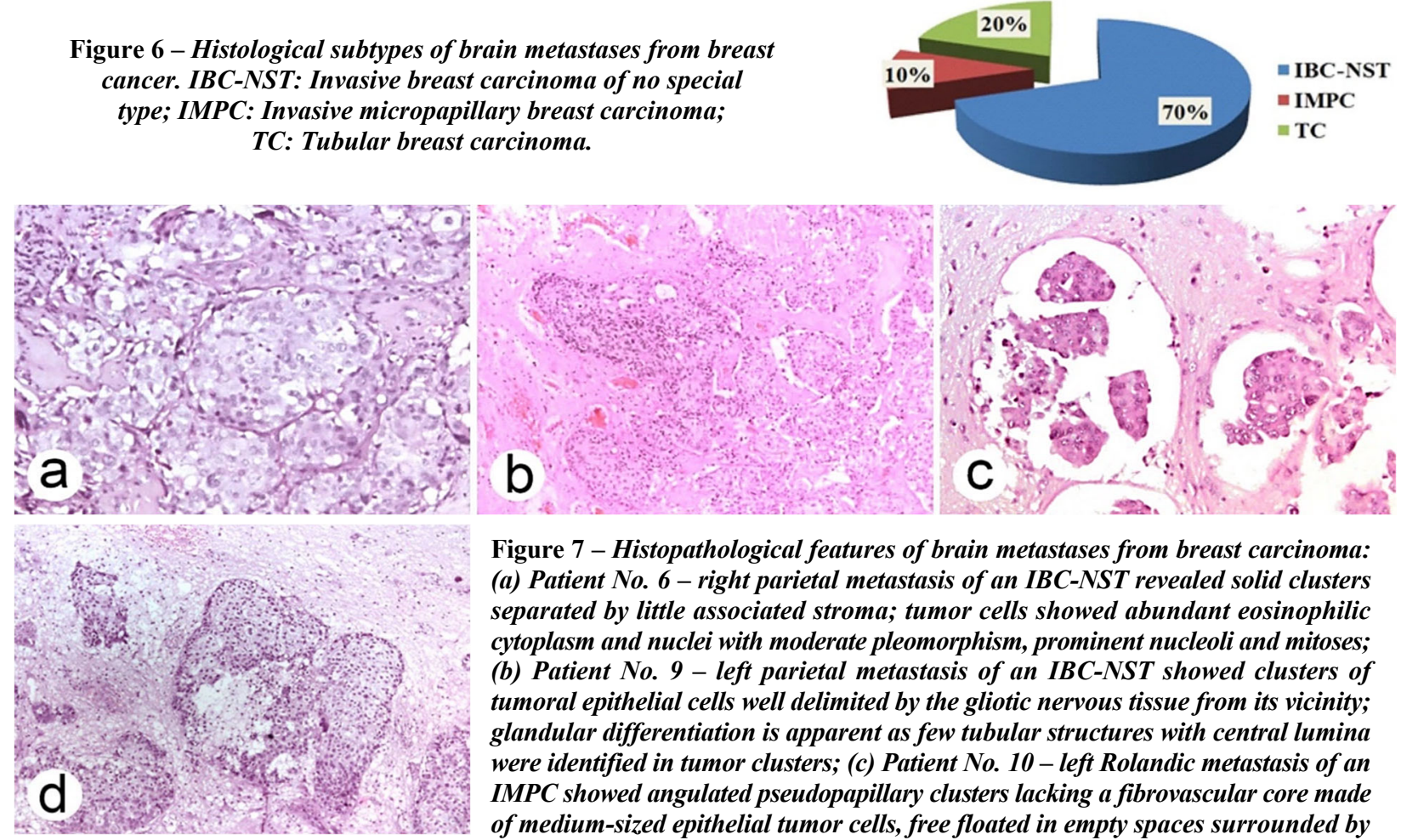

Figure 7 - Histopathological features of brain metastases from breast carcinoma: (a) Patient No. 6 - right parietal metastasis of an IBC-NST revealed solid clusters separated by little associated stroma; tumor cells showed abundant eosinophilic cytoplasm and nuclei with moderate pleomorphism, prominent nucleoli and mitoses; (b) Patient No. 9 - left parietal metastasis of an IBC-NST showed clusters of tumoral epithelial cells well delimited by the gliotic nervous tissue from its vicinity; glandular differentiation is apparent as few tubular structures with central lumina were identified in tumor clusters; (c) Patient No. 10 - left Rolandic metastasis of an IMPC showed angulated pseudopapillary clusters lacking a fibrovascular core made of medium-sized epithelial tumor cells, free floated in empty spaces surrounded by nervous glial tissue; (d) Patient No. 2 - right parietal metastasis of an IBC-NST showed a tumor that was well separated from the surrounding gliotic brain tissue and was made up of clusters of pleomorphic epithelial cells with well-defined limits and clear cytoplasm, similar with a "vegetaloid" appearance; some of the tumor nests revealed areas of comedo-type necrosis. HE staining: (a) $\times 400$; (b and d) $\times 100$; (c) $\times 200$. HE: Hematoxylin-Eosin; IBC-NST: Invasive breast carcinoma of no special type; IMPC: Invasive micropapillary breast carcinoma.

Figure 8 - Correlation between location and histological subtypes of brain metastases from breast cancer. IBC-NST: Invasive breast carcinoma of no special type; IMPC: Invasive micropapillary breast carcinoma; TC: Tubular breast carcinoma.
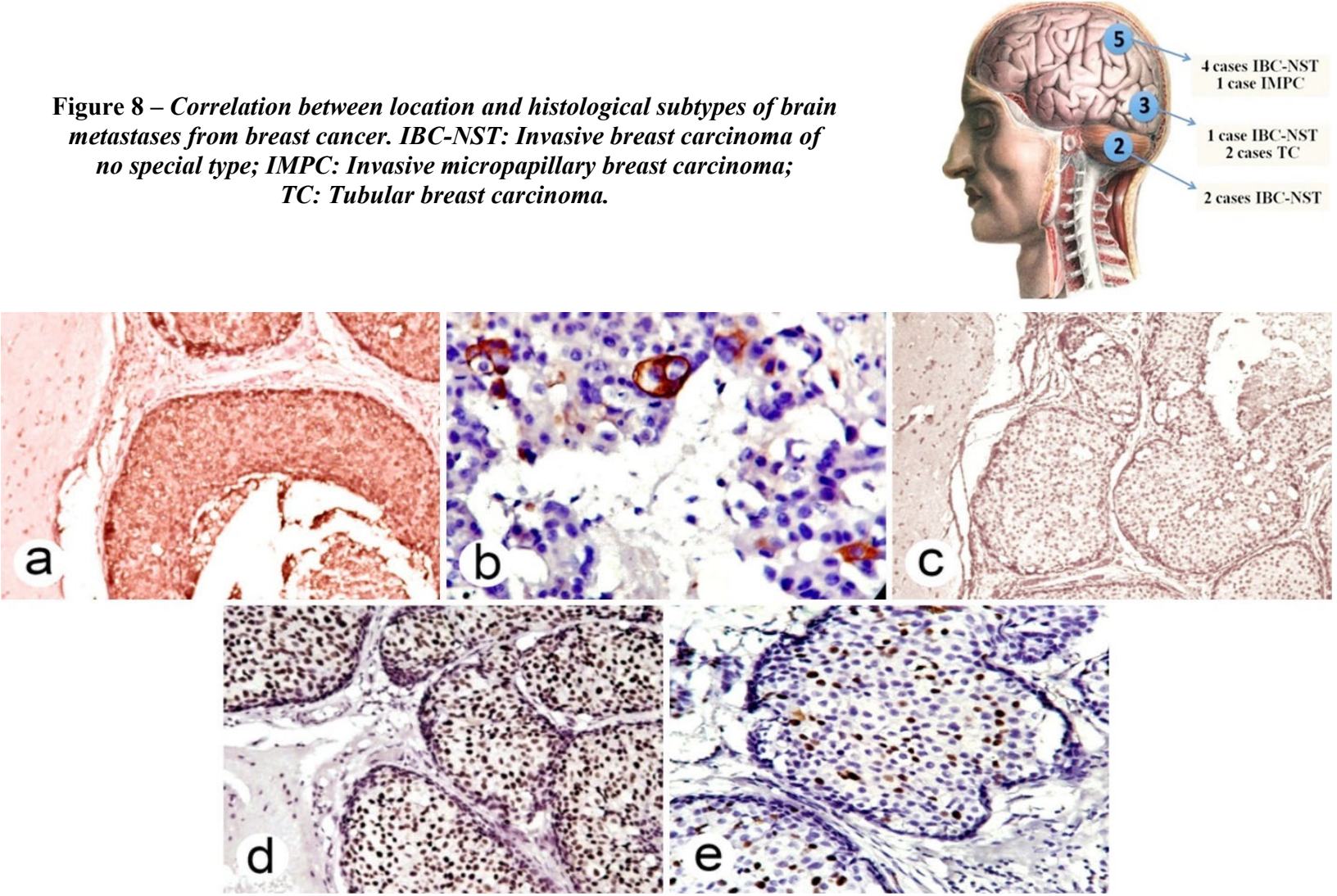

Figure 9 - Immunohistochemistry of invasive breast carcinoma of no special type: (a) Strong cytoplasmic positivity for anti-mammaglobin antibody (×100); (b) Focal strong cytoplasmic immunopositivity for anti-CK5/6 antibody ( $\times 400)$; (c) Negativity for anti-PR antibody in all tumoral cells and in the surrounding nervous tissue (×100); (d) Strong nuclear positivity for anti-ER antibody ( $\times 200)$; (e) Strong nuclear expression for anti-Ki67 antibody, with $16 \%$ Ki67 LI $(\times 400)$. CK56: Cytokeratin 5/6; ER: Estrogen receptor; LI: Labeling index; PR: Progesterone receptor. 


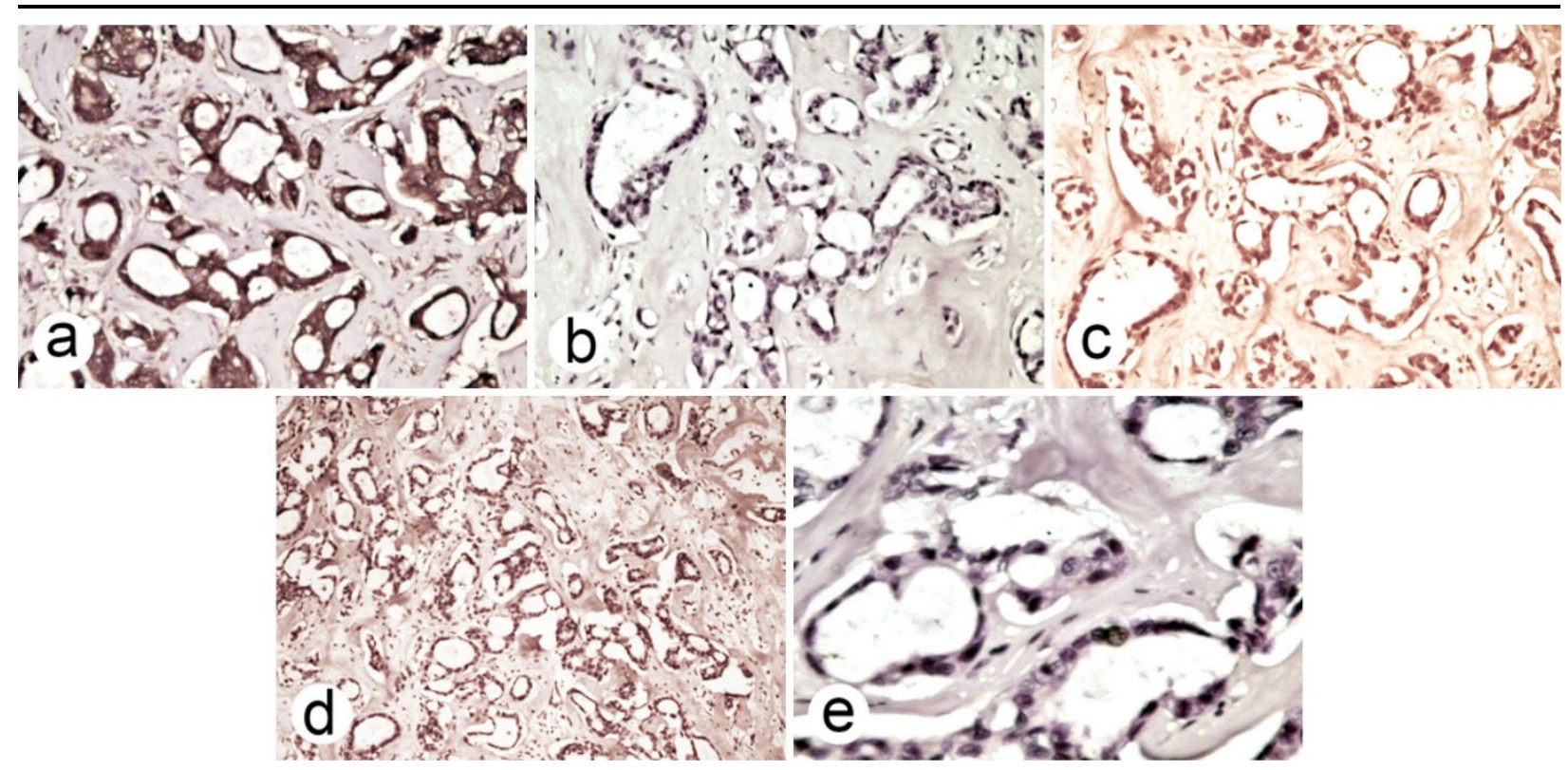

Figure 10 - Immunohistochemistry of tubular breast carcinoma: (a) Strong cytoplasmic positivity for anti-mammaglobin antibody ( $\times 200)$; (b) Negativity for anti-CK5/6 antibody in all tumor cells $(\times 200)$; (c) Negativity for anti-PR antibody in all tumor cells and in the surrounding nervous tissue ( $\times 100)$; (d) Negativity for anti-ER antibody $(\times 200)$; (e) Strong nuclear expression for anti-Ki67 antibody, with 3\% Ki67 LI (×400). CK56: Cytokeratin 5/6; ER: Estrogen receptor; LI: Labeling index; PR: Progesterone receptor.

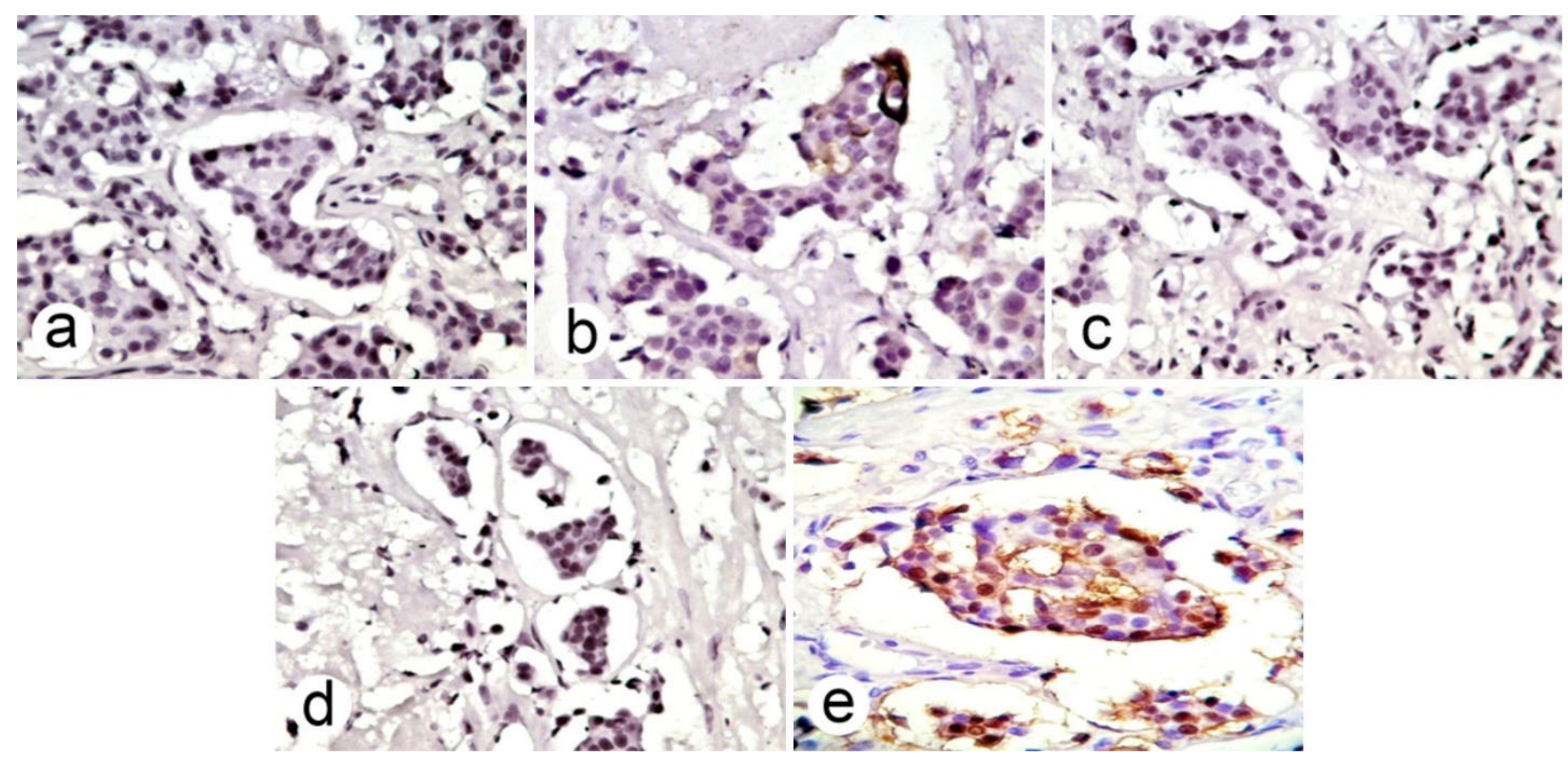

Figure 11 - Immunohistochemistry of invasive micropapillary breast carcinoma: (a) Negativity for anti-mammaglobin antibody (×400); (b) Strong focal cytoplasmic positivity for anti-CK5/6 antibody in all tumor cells (×400); (c) Negativity for anti-PR antibody in all tumor cells and in the surrounding nervous tissue (×400); (d) Negativity for anti-ER antibody (×400); (e) Strong nuclear expression for anti-Ki67 antibody, with 28\% Ki67 LI (×400). CK56: Cytokeratin 5/6; ER: Estrogen receptor; LI: Labeling index; PR: Progesterone receptor.

\section{口 Discussions}

The brain is the site of metastases originating in almost all organs of the human body, although some cancers with particular locations, such as mesothelioma [8], or colorectal carcinomas [9] are very rare at this level, but others, such as those originating in the lung or breast, are frequently diagnosed in this location.

Literature reported that the mean age of the patients at the time of $\mathrm{BC}$ diagnosis is 47 years. The average latency between the diagnosis of $\mathrm{BC}$ and the detection of $\mathrm{BMs}$ of $\mathrm{BC}$ is $2-3$ years. However, BMs can occur even 20 years after the diagnosis of BC [10]. Andreas Stark mentioned that $\mathrm{BMs}$ from $\mathrm{BC}$ are located more frequently in the supratentorial area than in the infratentorial space [10] and we identified the same distribution in our study.

BMs are associated with a poor prognosis because they cause sensory and cognitive neurological deficits [11]. The symptoms caused by the BMs depend on the exact size and location of the lesion. In cases with small tumors, neurological deficits are more obvious if the lesion is located in/near to eloquent brain areas. Large $\mathrm{BMs}$ can cause an increased intracranial pressure due to the blockage of the cerebrospinal fluid (CSF) pathways. 
The most common symptoms when a patient with a BM from a $\mathrm{BC}$ is presenting to the hospital may be ataxia $(23 \%)$, headache $(21 \%)$, visual disturbances $(15 \%)$, hemiparesis $(11 \%)$ and vertigo (11\%) [10].

In our study, one third of all patients were diagnosed with $\mathrm{BM}$ without a known $\mathrm{BC}$, in these cases the brain being the primary site of metastasis. Although the breast is much more visible to both the patient and the doctor than the colon, it seems that patients do not see a doctor for early detection of their BC. A recent study showed that in the case of colorectal cancers (CRCs), more common in male patients, only $7.4 \%$ of patients admitted to a neurosurgery clinic for excision of their metastatic brain tumors presented neurological symptoms without a prior diagnosis of a CRC [9]. These data mean that early BC screening programs need to be implemented in Romania as quickly as possible.

In our study, we noticed that surgery was performed to excise metastases located especially in functionally important areas, such as "eloquent brain areas", which are nervous regions that in case of an injury can result in loss of function [12], or in cerebellum, which is a major motor structure [13].

In the report published by Chow et al., the mean time to develop BM from a BC was 29 months. The authors found that patients who developed $\mathrm{BMs}$ had $\mathrm{BC}$ with a high HP grade, a more advanced tumor (T)-stage and lymph node (N)-stage, an ER-, PR-, and c-erbB-2+ status [14].

In a recent review, Rostami et al. (2016) analyzed 106 articles, which have been published over the past three decades and included 14599 patients with BMs from BC. These authors concluded that the median time between diagnosis of primary tumor and diagnosis of BM is 34 months. More than half $(54.2 \%)$ of the patients had more than two intracranial metastases, most of them being supratentorial (33\%), the cerebellar ones representing $16 \%$ [1].

In our study, we found that four-fifths of the BM from $\mathrm{BC}$ were located in the supratentorial region, affecting the parietal and occipital lobes, the rest being identified in the cerebellum. According to literature, there is a relationship between brain anatomy and BM location. Recently, Wang et al. (2019) [15] analyzed 335 patients with BMs from lung cancer and found out that this type of cancer metastasized mainly in the cerebellum $(56 \%)$, right parietal lobe $(54 \%)$, right frontal lobe (47\%), and left frontal lobe $(45 \%)$.

The spatial distribution of $\mathrm{BMs}$ from $\mathrm{BC}$, according to their HP subtypes, showed that TC developed in the occipital lobe, IMPC was diagnosed in the parietal lobe, but IBC-NST could be found in parietal lobe, occipital lobe, and cerebellum.

As in the study by Kyeong et al. in 2017 [16], in our study BMs from $\mathrm{BC}$ tend to have a certain spatial distribution, with preferential involvement of the parietal and occipital lobes, as well as the cerebellum, an aspect that can be related to the biological characteristics of different HP and molecular subtypes. Compared to the mentioned study, our research did not identify any case located in the frontal lobe, the limbic region, but the number of cases was limited, and we analyzed only the location of metastases that were excised, often the patient presenting more than two BMs. The above-mentioned authors [16] concluded in their article published in 2017 that the spatial distribution of BM is depending on the genetic composition of primary $\mathrm{BC}$ as TNBC can metastasize anywhere in the brain and human epidermal growth factor receptor 2 (HER2) subtypes positive and Luminal subtype is preferentially located in the territories irrigated by the posterior circulation, namely the occipital and cerebellum lobes.

Factors that increase the risk of developing a BM originating in a $\mathrm{BC}$ are young age ( $<35$ years old), premenopausal status, poorly differentiated tumors (highly proliferative, and genomically unstable tumors), the negative status of hormone receptors (HRs) (ER- and PR- on immunohistochemistry), and higher number of non-central nervous system (CNS) metastatic sites [11, 17].

Although many studies considered that young patients ( $<35$ years old) with $\mathrm{BC}$ have a particular risk of developing BMs [11, 17, 18], our patients were mostly in their seventh decade of life, and this feature could be particular for the $\mathrm{BMs}$ originating in $\mathrm{BC}$ diagnosed in our region.

Overall diagnostic accuracy of intra-operative smear technique achieved in the present study was $100 \%$, which proves the advantages of this examination: relatively high speed, simplicity, and effectiveness of diagnosis. This technique is extremely useful in neurosurgery for excision of BMs located in important functional areas of the brain.

In our study, the same with other researches, IBC-NST was the most common HP subtype in BM originating in $\mathrm{BC}$, but this fact could be correlated with the higher frequency of this HP type in the primary tumor, because most patients had invasive ductal histopathology (94\%) and grade 3 tumors (72\%) [19].

The $5^{\text {th }}$ edition $W H O$ Classification of Tumors of the Breast, published in December 2019 [7], reclassifies breast tumors and introduces new subtypes, while removing others. Regarding epithelial tumors of the breast, the group including invasive $\mathrm{BC}$ is maintained, but new subtypes are introduced.

Histologically, BM originating in BC from our study was well delineated by the surrounding gliotic nerve tissue, and mainly expressed the phenotype of IBC-NST, but rarer phenotypes such as TC and IMPC were also identified. We did not identify any case with an infiltrating lobular carcinoma (ILC). However, literature also admits that ILC is less likely than IBC-NST to be associated with BMs [20].

Hamilton et al. (2004) analyzed the demographic and pathological characteristics of patients with TC and compared them with those of patients with IBC-NST. They found that these patients were older at the time of diagnosis of their BM (63.9 years versus 59.6 years), the breast tumor evolved for a longer period of time until BM developed (87 months versus 34 months), and their survival after neurosurgical excision of $\mathrm{BM} / \mathrm{BMs}$ was longer compared to those with IBC-NST [21].

A 2020 article published by De Souza et al. reported the fact that the predominant HP type among the phenotypes of their series with BMs originating in BC was the IBCNST, which was diagnosed in $65.88 \%$ of cases [22], this value being similar to that obtained in our study. 
Regarding the prognosis of IMPC, which is a rare and new HP variant of BC, there is controversial data. Some authors claim that IMPC has a worse prognosis than IBCNST [23], but others affirm that there is no statistically significant difference between IMPC and IBC-NST, and therefore there is no need for proactive or radical clinical therapy [24].

Although in our series there is only one case of such a special type of $\mathrm{BC}$, the time from the diagnosis of the primary tumor is 27 months, a period almost similar to the mean time to the onset of a BM for the entire series and this fact supports the hypothesis that the prognosis of this subtype of BC is not different from that of IBC-NST. If until recently the traditional classification of $\mathrm{BC}$ was based on morphological aspects, in 2000 , considering that this classification system has certain limitations [25], a molecular classification of this type of cancer was proposed, which is still in the process of development and refining and represents the starting point for individualized therapies [26].

Based on molecular markers, $\mathrm{BC}$ can be classified into molecular subtypes [27, 28], as follows:

(1) Luminal type A is the most common subtype and is characterized by strong ER+ and/or PR+ immunostainings, but HER2-. From a HP point of view, it has a lower grade (low Ki67 LI) and is hormone responsive, having a good prognosis. Luminal type A disease generally requires only endocrine therapy.

(2) Luminal type B is characterized by ER+ and/or PR+ and HER2+ immunostainings. It correlates with tumors with higher grade (high Ki67 LI) and has a worse outcome compared to Luminal type A, but also like Luminal type A, this type B could be treated with endocrine therapy and chemotherapy.

(3) HER2+, but ER- is less common, being diagnosed at a younger age. Such cases present high-grade histopathology and an aggressive outcome, but this outcome could be improved with Herceptin.

(4) Basal-like type BC or TNBC is an aggressive subtype that is characterized by high grade histopathology, propensity for metastasis, and worse prognosis. Young females are at risk to develop this subtype of BC. Targeted therapies are currently under investigation, but chemotherapy seems to be indicated for most patients.

(5) Triple-positive $\mathrm{BC}$ with ER+/PR+ and HER2+ is a new subtype, which can be treated with anti-HER2 agents and chemotherapy [29].

In the study published by Laakmann et al., in 2019, $16 \%$ of $\mathrm{BC}$ patients treated with neo-adjuvant chemotherapy were diagnosed with metastatic disease after a median monitoring time of five years. Only a small percent $(3 \%)$ of patients were identified with BMs as the first metastatic site and factors significantly associated with this rare event were higher tumor stage before therapy, the presence of lymph node metastases, and HER $2+$ or triple-negative subtypes [30].

In another study, Gonzalez-Angulo \& Hortobagyi (2010) also found a small percentage (5\%) of patients who have developed BMs as the first site of metastatic disease. Negative HR status, nuclear grade 3, four or more positive lymph nodes and higher stage of the disease were factors considered to be associated with short time to BM in their series [31].
Kwon et al. (2010) found that the time period from the initial $\mathrm{BC}$ to the onset of $\mathrm{BM}$ was 52.9, 33.6, and 25.5 months for patients with HR+, HER2+, and triple receptor negative tumors [32], which can probably be extrapolated in our study, because there is a link between the presence of these molecular markers and the evolution of $\mathrm{BC}$ in our patients. The prognostic factors involved in the development of $\mathrm{BM}$ originating in $\mathrm{BC}$ are tumor phenotype (HER2, ER status, HP grade, proliferative rate), the degree of the extension of the disease outside the brain (the presence of lung, bone and/or BMs), patient's age, as well as patient performance status [33, 34]. The literature shows that young age, negative ER status, Ki67 $\mathrm{LI}$ are independent risk factors for the development of BM originating in $\mathrm{BC}$ [6]. A research group from Pakistan reported that ER positivity was visible in $87 \%$ of their cases of IMPC, but only $60 \%$ of IBC-NST showed ER positivity [35]. Another study, realized by some American researchers, showed that ER positivity ranged from $19.4 \%$ to $90.6 \%$ in IMPC [36]. We also found a strong, but focal cytoplasmic expression of basal CK5/6 only in $20 \%$ of $\mathrm{BMs}$ originating in invasive $\mathrm{BC}$ and/or IMPC. Positive CK5/6 (basal marker) expression was noted in 8\% (12) cases of TNBC. As such, CK5/6 could serve as poor prognostic marker in TNBC [6].

There are some studies regarding the impact of depression on the overall survival (OS) of these patients. Using self-rating depression scale (SDS) questionnaires, Guo et al. (2017) analyzed the correlation between HR status and depressive symptoms in patients with metastatic BC [37]. They found no significant differences regarding median OS between the depressed and non-depressed patient groups, both groups having a median OS of approximately 20 months. However, these authors identified a statistically significant reduction in median OS for HR-positive BC patients, with a median OS of 25 months for those with depressive symptoms and 32 months for those without depression. In contrast, the depressive status did not affect OS in HR-negative BC, as groups of patients, i.e., those with depression and those without depression had a median OS of about 14 months.

\section{ㅁ Conclusions}

Our study revealed the fact that BMs originating in BC show heterogeneity of HR status, although morphologically there is not so much diversity. In our study, the risk for the development of BMs seems to be correlated with the age over 50 years, BMs highlighting a negative status of $\mathrm{PR}$ and ER. We also found a very variable Ki67 LI, which correlated especially with the morphological subtype. We consider that our data could be useful for oncologists to apply some preventive or therapeutic measures.

\section{Conflict of interests}

The authors declare that they have no conflict of interests.

\section{References}

[1] Rostami R, Mittal S, Rostami P, Tavassoli F, Jabbari B. Brain metastasis in breast cancer: a comprehensive literature review. J Neurooncol, 2016, 127(3):407-414. https://doi.org/10.1007/ s11060-016-2075-3 PMID: 26909695 
[2] Lin NU, Claus E, Sohl J, Razzak AR, Arnaout A, Winer EP Sites of distant recurrence and clinical outcomes in patients with metastatic triple-negative breast cancer: high incidence of central nervous system metastases. Cancer, 2008, 113(10): 2638-2645. https://doi.org/10.1002/cncr.23930 PMID: 18833576 PMCID: PMC2835546

[3] Ghoncheh M, Pournamdar Z, Salehiniya H. Incidence and mortality and epidemiology of breast cancer in the world. Asian Pac J Cancer Prev, 2016, 17(S3):43-46. https://doi.org/ 10.7314/apjcp.2016.17.s3.43 PMID: 27165206

[4] World Health Organization (WHO). Romania. Cancer Country Profiles, 2014. https://www.who.int/cancer/country-profiles/ rou_en.pdf?ua=1

[5] Lin NU, Bellon JR, Winer EP. CNS metastases in breast cancer. J Clin Oncol, 2004, 22(17):3608-3617. https://doi.org/10.1200/ JCO.2004.01.175 PMID: 15337811

[6] Sim HW, Morris PG, Patil S, Khasraw M. Brain metastases in breast cancer. Expert Rev Anticancer Ther, 2014, 14(2):173183. https://doi.org/10.1586/14737140.2014.863468 PMID: 24308683

[7] Tan PH, Ellis I, Allison K, Brogi E, Fox SB, Lakhani S, Lazar AJ Morris EA, Sahin A, Salgado R, Sapino A, Sasano H, Schnitt S, Sotiriou C, van Diest P, White VA, Lokuhetty D, Cree IA; WHO Classification of Tumours Editorial Board. The 2019 World Health Organization classification of tumours of the breast. Histopathology, 2020, 77(2):181-185. https://doi.org/10.1111/ his.14091 PMID: 32056259

[8] Marzullo A, Scattone A, Rossi R, Cimmino A, Punzi A, Corsi F, Cavone D, Lettini T, Serio G. Malignant pleural mesothelioma presenting with symptomatic brain metastases: report of a case. Rom J Morphol Embryol, 2013, 54(3):649-653. PMID: 24068419

[9] Scripcariu V, Ciobanu Apostol DG, Dumitrescu GF, Turliuc MD, Sava A. Clinical, histopathological and immunohistochemical features of brain metastases originating in colorectal cancer: a series of 27 consecutive cases. Rom J Morphol Embryol, 2020, 61(1):81-93. https://doi.org/10.47162/RJME.61.1.09 PMID: 32747898 PMCID: PMC7728123

[10] Stark AM. Neurosurgical treatment of breast cancer metastases to the neurocranium. Patholog Res Int, 2010, 2011:549847. https://doi.org/10.4061/2011/549847 PMID: 21209717 PMCID: PMC3010687

[11] Witzel I, Oliveira-Ferrer L, Pantel K, Müller V, Wikman H. Breast cancer brain metastases: biology and new clinical perspectives. Breast Cancer Res, 2016, 18(1):8. https://doi. org/10.1186/s13058-015-0665-1 PMID: 26781299 PMCID: PMC4717619

[12] Zakaria J, Prabhu VC. Cortical mapping in the resection of malignant cerebral gliomas. In: De Vleeschouwer S (ed). Glioblastoma [Internet]. Codon Publications, Brisbane (AU), 2017 Sep 27, Chapter 13. https://doi.org/10.15586/codon. glioblastoma.2017.ch13 PMID: 29251868 Bookshelf ID: NBK470008

[13] Mottolese C, Richard N, Harquel S, Szathmari A, Sirigu A, Desmurget M. Mapping motor representations in the human cerebellum. Brain, 2013, 136(Pt 1):330-342. https://doi.org/ 10.1093/brain/aws186 PMID: 22945964

[14] Chow L, Suen D, Ma KK, Kwong A. Identifying risk factors for brain metastasis in breast cancer patients: implication for a vigorous surveillance program. Asian J Surg, 2015, 38(4): 220-223. https://doi.org/10.1016/j.asjsur.2015.03.003 PMID: 26216259

[15] Wang G, Xu J, Qi Y, Xiu J, Li R, Han M. Distribution of brain metastasis from lung cancer. Cancer Manag Res, 2019, 11: 9331-9338. https://doi.org/10.2147/CMAR.S222920 PMID: 31802951 PMCID: PMC6830371

[16] Kyeong S, Cha YJ, Ahn SG, Suh SH, Son EJ, Ahn SJ. Subtypes of breast cancer show different spatial distributions of brain metastases. PLoS One, 2017, 12(11):e0188542. https:// doi.org/10.1371/journal.pone.0188542 PMID: 29155879 PMCID: PMC5695816

[17] Lee KL, Chen G, Chen TY, Kuo YC, Su YK. Effects of cancer stem cells in triple-negative breast cancer and brain metastasis: challenges and solutions. Cancers (Basel), 2020, 12(8):2122. https://doi.org/10.3390/cancers12082122 PMID: 32751846 PMCID: PMC7463650

[18] Hung MH, Liu CY, Shiau CY, Hsu CY, Tsai YF, Wang YL, Tai LC, King KL, Chao TC, Chiu JH, Su CH, Lo SS, Tzeng CH,
Shyr YM, Tseng LM. Effect of age and biological subtype on the risk and timing of brain metastasis in breast cancer patients. PLoS One, 2014, 9(2):e89389. https://doi.org/10. 1371/journal.pone.0089389 PMID: 24586742 PMCID: PMC 3933537

[19] Shen Q, Sahin AA, Hess KR, Suki D, Aldape KD, Sawaya R, Ibrahim NK. Breast cancer with brain metastases: clinicopathologic features, survival, and paired biomarker analysis. Oncologist, 2015, 20(5):466-473. https://doi.org/10.1634/the oncologist.2014-0107 PMID: 25802405 PMCID: PMC4425375

[20] Tham YL, Sexton K, Kramer R, Hilsenbeck S, Elledge R. Primary breast cancer phenotypes associated with propensity for central nervous system metastases. Cancer, 2006, 107(4): 696-704. https://doi.org/10.1002/cncr.22041 PMID: 16826579

[21] Hamilton LJ, Evans AJ, Pinder SE, James JJ, Gutteridge E, Cornford EJ, Burrell HC, Chan SY, Robertson JF, Cheung KL. Metastatic carcinoma of the breast with tubular features: differences compared with metastatic ductal carcinoma of no specific type. Clin Oncol (R Coll Radiol), 2004, 16(2):119-124. https://doi.org/10.1016/j.clon.2003.11.007 PMID: 15074735

[22] de Souza GMR, Carvalho AF, Santiago SF, Pinho MAMR, Ramadan DR, Tufik S, Feres MC. Main phenotypes and histological types of breast cancer in young women attended at a reference hospital in women's health in the city of São Paulo. Breast Cancer Manag, 2020, 9(4). https://doi.org/10. 2217/bmt-2020-0021

[23] Yu JI, Choi DH, Park W, Huh SJ, Cho EY, Lim YH, Ahn JS, Yang JH, Nam SJ. Differences in prognostic factors and patterns of failure between invasive micropapillary carcinoma and invasive ductal carcinoma of the breast: matched casecontrol study. Breast, 2010, 19(3):231-237. https://doi.org/ 10.1016/j.breast.2010.01.020 PMID: 20304650

[24] Hao S, Zhao YY, Peng JJ, Ren F, Yang WT, Yu KD, Shao ZM. Invasive micropapillary carcinoma of the breast had no difference in prognosis compared with invasive ductal carcinoma: a propensity-matched analysis. Sci Rep, 2019, 9(1):286. https://doi.org/10.1038/s41598-018-36362-8 PMID: 30670771 PMCID: PMC6343026

[25] Perou CM, Sørlie T, Eisen MB, van de Rijn M, Jeffrey SS, Rees CA, Pollack JR, Ross DT, Johnsen H, Akslen LA, Fluge O, Pergamenschikov A, Williams C, Zhu SX, Lønning PE, Børresen-Dale AL, Brown PO, Botstein D. Molecular portraits of human breast tumours. Nature, 2000, 406(6797):747-752. https://doi.org/10.1038/35021093 PMID: 10963602

[26] Eliyatkın N, Yalçın E, Zengel B, Aktaş S, Vardar E. Molecular classification of breast carcinoma: from traditional, old-fashioned way to a new age, and a new way. J Breast Health, 2015, 11(2):59-66. https://doi.org/10.5152/tjbh.2015.1669 PMID: 28331693 PMCID: PMC5351488

[27] Goldhirsch A, Wood WC, Coates AS, Gelber RD, Thürlimann B, Senn HJ; Panel members. Strategies for subtypes - dealing with the diversity of breast cancer: highlights of the St. Gallen International Expert Consensus on the primary therapy of early breast cancer 2011. Ann Oncol, 2011, 22(8):1736-1747. https://doi.org/10.1093/annonc/mdr304 PMID: 21709140 PMCID: PMC3144634

[28] Al-Thoubaity FK. Molecular classification of breast cancer: a retrospective cohort study. Ann Med Surg (Lond), 2019, 49: 44-48. https://doi.org/10.1016/j.amsu.2019.11.021 PMID: 31890196 PMCID: PMC6926136

[29] lancu G, Vasile D, lancu RC, Daviţoiu DV. "Triple positive" breast cancer - a novel category? Rom J Morphol Embryol, 2017, 58(1):21-26. PMID: 28523293

[30] Laakmann E, Witzel I, Fasching PA, Rezai M, Schem C, Solbach $C$, Tesch $H$, Klare $P$, Schneeweiss A, Salat C, Zahm DM, Blohmer JU, Ingold-Heppner B, Huober J, Hanusch C, Jackisch C, Reinisch M, Untch M, von Minckwitz G, Nekljudova V, Müller V, Loibl S. Development of central nervous system metastases as a first site of metastatic disease in breast cancer patients treated in the neoadjuvant trials GeparQuinto and GeparSixto. Breast Cancer Res, 2019, 21(1):60. https://doi.org/10.1186/s13058-019-1144-x PMID: 31077239 PMCID: PMC6509843

[31] Gonzales-Angulo AM, Hortobagyi GN. Brain metastases and breast cancer subtypes, Onkologie, 2010, 33(4):143-144. https://doi.org/10.1159/000296307 PMID: 20389139

[32] Kwon HC, Oh SY, Kim SH, Lee S, Kwon KA, Lee JH, Lee MR, Cho SH, Choi YJ, Kim HJ. Clinical outcomes in patients with 
triple-negative breast cancer and brain metastases. J Breast Cancer, 2010, 13(2):160-166. https://doi.org/10.4048/jbc.2010. 13.2.160

[33] Stelzer KJ. Epidemiology and prognosis of brain metastases. Surg Neurol Int, 2013, 4(Suppl 4):S192-S202. https://doi.org/ 10.4103/2152-7806.111296 PMID: 23717790 PMCID: PMC 3656565

[34] Koniali L, Hadjisavvas A, Constantinidou A, Christodoulou K, Christou Y, Demetriou C, Panayides AS, Pitris C, Pattichis CS, Zamba-Papanicolaou E, Kyriacou K. Risk factors for breast cancer brain metastases: a systematic review. Oncotarget, 2020, 11(6):650-669. https://doi.org/10.18632/oncotarget.27453 PMID: 32110283 PMCID: PMC7021234

[35] Hashmi AA, Aijaz S, Mahboob R, Khan SM, Irfan M, Iftikhar N, Nisar M, Siddiqui M, Edhi MM, Faridi N, Khan A. Clinicopathologic features of invasive metaplastic and micropapillary breast carcinoma: comparison with invasive ductal carcinoma of breast. BMC Res Notes, 2018, 11(1):531. https://doi.org/ 10.1186/s13104-018-3623-z PMID: 30064485 PMCID: PMC 6069771

[36] Chen AC, Paulino AC, Schwartz MR, Rodriguez AA, Bass BL, Chang JC, Teh BS. Population-based comparison of prognostic factors in invasive micropapillary and invasive ductal carcinoma of the breast. Br J Cancer, 2014, 111(3):619-622. https:// doi.org/10.1038/bjc.2014.301 PMID: 24921921 PMCID: PMC 4119976

[37] Guo X, Xu J, Ying E, Yu Z, Sun T. Correlation between hormone receptor status and depressive symptoms in patients with metastatic breast cancer, Oncotarget, 2017, 8(31):5077450781. https:doi.org/10.18632/oncotarget.15037 PMID: 28881602 PMCID: PMC5584203

\section{Corresponding authors}

Claudia Florida Costea, Associate Professor, MD, PhD, Department of Surgery II, Faculty of Medicine, Grigore T. Popa University of Medicine and Pharmacy, 16 Universităţii Street, 700115 laşi, Romania; Phone +40744-972 648, e-mail: costea10@yahoo.com

Ruxandra Vatavu, MD, PhD Student, Doctoral School, Grigore T. Popa University of Medicine and Pharmacy, 16 Universităţii Street, 700115 laşi, Romania; Phone +40762-205 570, e-mail: ruxandravatavu@yahoo.com

Received: April 18, 2021

Accepted: December 27, 2021 